\title{
Assessing the impact of medication use on trends in major coronary risk factors in older British men: a cohort study
}

\author{
Sarah L. Hardoon ${ }^{a}$, Peter H. Whincup ${ }^{b}$, S. Goya Wannamethee ${ }^{a}$, Lucy T. Lennona, Simon \\ Capewell' , and Richard W. Morris ${ }^{\mathrm{a}}$ \\ aDepartment of Primary Care and Population Health, Division of Population Health, UCL, London \\ bDivision of Community Health Sciences, St George's, University of London, London \\ 'School of Population Community and Behavioural Sciences, Division of Public Health, University \\ of Liverpool, Liverpool, UK
}

\begin{abstract}
Aims-To investigate the role of medication in 20-year trends in blood pressure (BP) and blood lipids in older British men.

Methods and results- $\mathrm{BP}$ and lipids were measured in 4231 men from a representative cohort at baseline (1978-1980, aged 40-59 years) and after 20 years (1998-2000). Cohort-wide ageadjusted 20-year mean changes were as follows: systolic BP $-7.6 \mathrm{mmHg}$ (95\% confidence interval: -9.7 to -5.4 ); diastolic $\mathrm{BP}+3.3 \mathrm{mmHg}(+2.2$ to +4.5$)$; non-high-density lipoprotein (HDL)-cholesterol $-0.4 \mathrm{mmol} / \mathrm{l}(-0.5$ to -0.2$)$; HDL-cholesterol $+0.16 \mathrm{mmol} / \mathrm{l}(+0.13$ to + 0.19). Much (79\%) of the systolic BP fall occurred only among 1561 men (37\%) reporting the use of BP-lowering medication during the follow-up; systolic BP changed by $-12.3 \mathrm{mmHg}$ ( -14.7 to $-9.9)$ and $-1.6 \mathrm{mmHg}(-3.7$ to +0.5$)$ among medication users and men not using medication, respectively $(P<0.001$ for medication-time interaction). One-third of the non-HDL-cholesterol fall occurred only among 302 men (8\%) reporting the use of lipid-regulating drugs; non-HDLcholesterol changed by $-1.8 \mathrm{mmol} / \mathrm{l}(-2.0$ to -1.6$)$ and $-0.2 \mathrm{mmol} / \mathrm{l}(-0.4$ to -0.1$)$ among medication users and men not using medication, respectively ( $P<0.001$ for interaction). The HDLcholesterol increase was not associated with lipid-regulating drug use ( $P=0.15$ for interaction).
\end{abstract}

Conclusion-Decreases in BP were largely confined to medication users and overall changes in non-HDL-cholesterol were modest, suggesting the need for greater efforts to reduce BP and cholesterol among the general population. HDL-cholesterol increased among all men, likely reflecting cohort-wide improvements in associated health behaviours.

\section{Keywords}

antihypertensive medication; blood pressure; cholesterol; epidemiology; lipid-regulating medication; time trends

\footnotetext{
(C) 2010 The European Society of Cardiology

Correspondence to Sarah L. Hardoon, MSc, Department of Primary Care and Population Health, Division of Population Health, UCL Medical School, Royal Free Campus, Rowland Hill Street, London NW3 2PF, UK Tel: + 442077940500 x34757; fax: + 44 2077941224; s.hardoon@ucl.ac.uk.

Conflict of interest: none declared.
} 


\section{Introduction}

Mortality from coronary heart disease (CHD) has been declining since the late 1970s in the United Kingdom [1], and the incidence of myocardial infarction has fallen by about half over the same period [2]. Changes in the major cardiovascular risk factors can account for a considerable proportion of the decline in incidence and mortality [2-5]. Although the most important single factor has been a fall in cigarette smoking, favourable trends in blood pressure (BP), high-density lipoprotein (HDL)-cholesterol and low-density lipoprotein (LDL)-cholesterol have also made contributions $[2,4,5]$. The reasons for the favourable changes in BP and blood lipids, however, have not been examined. The trends may reflect favourable changes in the underlying determinants of BP or blood lipids in the population; for BP adiposity, alcohol intake, physical activity and dietary factors (particularly salt intake) are known modifiable determinants [6] whereas for total and LDL-cholesterol dietary saturated fat intake, adiposity and physical activity are important [7]. Alternatively, the trends in these risk factors may reflect the increasingly widespread use of specific medications to lower BP (including particularly angiotensin-converting enzyme inhibitors, $\beta$-blockers, calcium channel blockers, diuretics) and to lower total and LDL-cholesterol (particularly statins) [8].

Understanding the contributions of medication-related and nonmedication-related factors to the changes in BP and blood lipids, which have occurred during the recent decades could help to inform efforts to bring about further reductions in the risk of cardiovascular disease, which continues to be the leading cause of death in the UK [1]. The aim of this study is therefore to assess the role of medication in changes in systolic BP (SBP), diastolic BP (DBP), non-HDL-cholesterol and HDL-cholesterol, which have occurred in older British men between 1978 and 2000. Trends in BP and blood lipids are examined separately in groups of men who received and did not receive BP-lowering or lipid-regulating medications. The British Regional Heart Study, a socially and geographically representative cohort of middle aged-men in Britain, was used for this analysis.

\section{Methods}

\section{British Regional Heart Study}

The British Regional Heart Study has been described in detail elsewhere [9]. A total of 7735 men were recruited between 1978 and 1980 from one general practice in each of 24 medium-sized towns across Britain, by random sampling, stratified by practice and 5-year age group. The men were aged 40-59 years when recruited and have been followed up for more than 25 years for cardiovascular outcomes and all-cause mortality.

\section{Assessment of main outcomes}

The main outcomes were SBP, DBP, HDL-cholesterol and non-HDL-cholesterol, measured at physical examinations at baseline (1978-1980) and after 20 years (1998-2000). BP was measured at baseline using the London School of Hygiene and Tropical Medicine sphygmomanometer and at the 20-year examination using the Dinamap 1846SX vital signs monitor (Critikon Inc., Tampa, Florida, USA). On both occasions, the mean of two successive readings, adjusted for observer variation, was used. The 20-year SBP measurements were adjusted for systematic overestimation of the Dinamap for SBP but not for DBP, identified in earlier studies [10]. At baseline, nonfasting blood samples were analysed for serum total cholesterol by a modified Liebermann-Burchard method on a Technicon SMA 12/60 analyser (Technicon Instruments, Tarrytown, New York, USA). At 20 years, fasting blood samples were analysed for serum total cholesterol using a Hitachi 747 automated analyzer (Hitachi, Tokyo, Japan). The Liebermann-Burchard method or 
enzymic procedures after precipitation with magnesium phosphotungstate were used to measure HDL-cholesterol. Cross-calibration of the two assays was done by remeasuring some baseline samples with the assay techniques used at 20 years. In 47 individuals, the mean within-person differences (remeasured minus baseline) in total cholesterol and in HDL-cholesterol were $0.067 \mathrm{mmol} / \mathrm{l}(\mathrm{SE} 0.105)(P=0.5)$ and $0.072 \mathrm{mmol} / \mathrm{l}(\mathrm{SE} 0.128)$ $(P=0.4)$, respectively. In view of the lack of significant evidence for systematic differences between the measurement methods, these between-assay differences were not taken into account in the main analysis. However, because the comparability of baseline and 20 year measurements is particularly important in this study, additional sensitivity analyses were conducted using baseline measurements adjusted for the small differences observed. NonHDL-cholesterol was computed as the difference between total cholesterol and HDLcholesterol, and would thus mainly represent LDL-cholesterol.

\section{Assessment of medication use}

The men completed an interview-administered questionnaire at baseline (1978-1980), a selfadministered questionnaire at 20 year's follow-up (1998-2000), and self-administered questionnaires at three intermediate time points at 5 year's follow-up (1983-1985), in 1992 and in 1996. From questions in each questionnaire about medication use, current use of drugs to lower BP or to regulate lipid levels at each time point was determined. The information at each time point was then combined to produce an indicator for BP-lowering drug use at any time between baseline and 20 years, and a similar indicator for lipidregulating drug use at any time between baseline and 20 years.

\section{Measurement of risk factors for hypertension and dyslipidemia}

Potential risk factors for high SBP and DBP considered in this analysis were body mass index (BMI), physical inactivity and alcohol intake. The risk factors considered for high non-HDL-cholesterol were BMI and physical inactivity. For low HDL-cholesterol, BMI, physical inactivity, alcohol use and smoking status were considered. Differences between medication users and non-medication users in the overall levels of these lifestyle factors, as well as in temporal changes in these factors could potentially confound estimates of the modifying effect of medication use on trends in BP and cholesterol. Therefore, recorded levels of each factor at both baseline and the 20-year follow-up were used in analyses. BMI was calculated from height and weight measurements taken at the two physical examinations. Identical questions in questionnaires at the two time points were used to ascertain smoking status, alcohol intake and physical activity. From the information given, men were categorized at each time point as 'current', 'ex' or 'never' smokers. The men's alcohol consumption was categorized as 'never,' 'occasionally,' 'light,' 'moderate' and 'heavy' [11]. Answers to questions relating to recreational activities, regular walking and cycling and sporting activity were combined to give each man a physical activity score, which has been validated [12]. Men were grouped into six categories based on their score as 'inactive,' 'occasional,' 'light,' 'moderate,' 'moderately vigorous' and 'vigorous.'

\section{Individuals included in the study}

All men who were alive at the 20-year follow-up and attended the 20-year physical examination were included in the analyses.

\section{Statistical methods}

Population-averaged age-adjusted changes in each of the four outcomes were estimated from linear regression of the outcome on a time variable (taking the value 0 at baseline, and value 20 at 20 years), adjusting for age. Generalized estimating equations with robust standard errors were used in the regressions to take account of the repeated measures of each outcome 
(one at baseline and a second at 20 years) for each man. An interaction term of time with an indicator for medication use at any time between baseline and 20 years was added to the model to estimate the trend in the outcome according to whether a man had received medication or not. $P$ values were obtained by means of Wald tests from the associated regression models. Predicted BP and cholesterol levels for a 60-year-old man at baseline and 20 years were estimated from the regression models. Multivariable models, adjusting additionally for the current levels at both time points of the risk factors for each outcome (BMI as a continuous variable and physical activity, smoking status and alcohol intake categorized as described above), were used to take account of possible differences in these factors between the medicated and unmedicated men. Analyses were performed using STATA (StataCorp LP, College Station, Texas, USA).

\section{Results}

Of 7735 men who entered the study, 4252 surviving men were reexamined at 20 years. Of these men, $21(0.5 \%)$ were missing a valid BP measurement at baseline and/or 20 years, leaving 4231 men for inclusion in analyses of trends in SBP and DBP. Three hundred and eighty-six men (9\%) were missing a valid lipid measurement at baseline and/or 20 years and a further four men $(0.09 \%)$ were missing records of lipid-regulating medication use, leaving 3862 men for inclusion in analyses of trends in non-HDL and HDL-cholesterol. A total of 1561 (37\%) out of 4231 men reported the use of BP-lowering drugs at some point over the 20 years; the proportion increased from $3.0 \%$ at baseline (1978-1980) to $33 \%$ at 20 years follow-up (1998-2000). Three hundred and two (7.8\%) out of 3862 men reported the use of lipid-regulating drugs at some point over the 20 years; none reported use at baseline, compared with $7.4 \%$ at 20 years.

Adjusting for age, mean SBP changed by $-7.56 \mathrm{mmHg}$ [95\% confidence interval (CI): 9.69 to $-5.43, P<0.001]$ whereas mean DBP changed by $+3.31 \mathrm{mmHg}(95 \% \mathrm{CI}:+2.18$ to $+4.45, P<0.001$ ) over 20 years (Table 1 ). The trends in SBP and DBP varied according to BP-lowering drug use. Mean changes in SBP were $-12.30 \mathrm{mmHg}$ (95\% CI: - 14.69 to 9.92) among medication users and $-1.61 \mathrm{mmHg}(95 \% \mathrm{CI}$ : -3.71 to +0.49$)$ among men not on medication $(P<0.001$ for medication-time interaction). Mean changes in DBP were $1.22 \mathrm{mmHg}(95 \% \mathrm{CI}:-2.51$ to +0.07$)$ among medication users and $+7.68 \mathrm{mmHg}(95 \% \mathrm{CI}$ : +6.57 to +8.79$)$ among men not on medication $(P<0.001$ for medication-time interaction).

Adjusting for age, mean non-HDL-cholesterol changed by $-0.35 \mathrm{mmol} / \mathrm{l}(95 \% \mathrm{CI}:-0.46$ to $-0.24, P<0.001)$. The change among medication users was $-1.78 \mathrm{mmol} / \mathrm{l}(95 \% \mathrm{CI}:-1.96$ to -1.60$)$, compared with a change of $-0.24 \mathrm{mmol} / \mathrm{l}(95 \% \mathrm{CI}$ : -0.35 to -0.13$)$ among men not on medication $(P<0.001$ for medication-time interaction). Mean HDL-cholesterol changed by $+0.16 \mathrm{mmol} / 1(95 \% \mathrm{CI}:+0.13$ to $+0.19, P<0.001)$. There was no evidence that the trend in HDL-cholesterol varied according to lipid-regulating drug use. HDL-cholesterol changed by $+0.18(95 \%$ CI: +0.14 to +0.23$)$ among medication users and by $+0.16(95 \%$ CI: +0.13 to +0.19$)$ among men not on medication $(P=0.15$ for medication-time interaction).

The results suggest that had none of the men using BP-lowering medication been present, we would have expected an overall average decline in SBP among all men of $1.61 \mathrm{mmHg}$ (the change among men not on medication). Given that the actual average decline observed was $7.56 \mathrm{mmHg}$, this suggests that $(7.56-1.61) / 7.56=79 \%$ of the overall cohort-wide decline in SBP may be attributed to the greater changes occurring among that select (high-risk) group of medication users, over and above the changes occurring among men not on medication. Similarly, $31 \%$ of the decline in non-HDL-cholesterol and none of the increase in HDL-cholesterol may be attributed to greater changes occurring only among lipid- 
regulating drug users, over and above the background changes occurring in men not on medication.

With additional adjustment for measured risk factors (BMI, alcohol use and physical activity for SBP and DBP; BMI and physical activity for non-HDL-cholesterol; BMI, physical activity, smoking and alcohol use for HDL-cholesterol), the favourable trends in SBP, nonHDL-cholesterol and HDL-cholesterol became slightly stronger and the unfavourable trend in DBP became weaker, but the interactions between medication use and time were essentially unchanged, reflecting the little variation in the absolute levels and temporal changes in these behavioural risk factors according to BP-lowering medication use and lipid-regulating medication use. In sensitivity analyses adjusted for the (nonsignificant) systematic differences in lipid measurements between the baseline and 20-year assay techniques described in the Methods section, the trends in non-HDL-cholesterol were unchanged and, although the increase in HDL became slightly weaker, it remained significant and there remained no difference according to medication use.

\section{Discussion}

\section{Summary of main findings}

Over a 20-year period between 1978-1980 and 1998-2000, a significant favourable increase of $14 \%$ in average HDL-cholesterol, and significant but more modest favourable falls of 5 and $7 \%$ in SBP and non-HDL-cholesterol, respectively, were observed in this representative survivor cohort of British men. Most (79\%) of the fall in SBP and a smaller proportion (31\%) of the fall in non-HDL-cholesterol could be attributed to larger decreases in medication users over and above the decreases among men not on medication. The favourable increase in HDL-cholesterol, however, was independent of medication use. DBP did not change favourably over the 20 years.

\section{Strengths and limitations}

This study provided reliable data on long-term changes in BP and cholesterol in a socially and geographically representative sample of older British men. Cross-calibration of BP and lipid measurement techniques used at 20 years with those at baseline ensured comparability between the two time points. The use of self-report to identify medication needs consideration. Repeating questionnaires between baseline and the 20-year follow-up helped to ensure that all users of relevant medications over this period were identified, as did asking in the questionnaires not only about specific medications but to list all prescriptions. Data on population-wide prevalence of medication use is limited, particularly in earlier years. Findings from routine prescription records showed that in 1998, among men aged 65-74 years, the prevalence of prescribing of lipid-regulating medication was $7.8 \%$ [13], a figure that agrees closely with our data (7.7\% in 1998-2000), which helps to validate our selfreported medication measure. Corresponding figures for blood pressure medication from this report could not be compared as patients may be prescribed more than one type of blood pressure medication, leading to larger prescription numbers. However, self-report of antihypertensive medication has been shown to agree reasonably closely with prescription data [14]. Moreover, self-report is arguably a better reflection of medication compliance than prescription records.

The analyses were necessarily based on those surviving men who attended the 20-year follow-up reexamination. This could introduce survival and response biases. The most likely impact would be overestimation of the favourable trends observed, as survivors tend to be 'healthier'. However, importantly, it is unlikely to explain the differential trends according to medication use and thus the role of medication, which is the key finding in this study. To 
examine the potential survivor effect in this cohort, baseline levels of blood pressure and cholesterol among those who survived to attend the 20-year examination were compared with levels among nonattendees [15]. The mean differences between the baseline levels were small to negligible (mean differences of $0 \mathrm{mmol} / \mathrm{l}, 2.4$ and $1.2 \mathrm{mmHg}$ for total cholesterol, SBP and DBP respectively), especially when compared with the overall changes over time, suggesting that the selection was unlikely to have had a dramatic influence on the observed trends. As might be expected, baseline levels of SBP, DBP, and non-HDLcholesterol were much higher in those men who reported subsequent medication use (Table 1), thus there was arguably greater potential for a decline in these groups. Nevertheless, in separate analyses, including baseline levels of the outcome as a covariate to adjust for regression to the mean [16], the changes in SBP, DBP and non-HDL-cholesterol in the medication users remained significantly greater than the changes in men not using medication while there remained no significant difference in the HDL-cholesterol trends. Only $8 \%$ of men were on lipid-regulating medications, raising issues of low statistical power (wide confidence intervals). The cohort comprises older British men, for whom prevalence of medication use is higher than for younger men and women and therefore generalizability of these results to other populations is uncertain. In particular, if prevalence of medication use is lower in another population, the percentage of the BP and cholesterol trends attributable to added changes among medication users will be lower.

\section{Comparison with other studies}

The overall decline in SBP is consistent with cross-sectional routine data for England reported in the Health Survey for England 1998 [17] and with SBP trends observed in Glasgow, Scotland (WHO MONICA [18,19]). However, modest declines in DBP were also reported in these two studies, in contrast to our observed increase. Data on long-term trends in cholesterol in the UK is limited, particularly for HDL-cholesterol, although a separate Health Survey for England report on cardiovascular disease and risk factors [20] highlighted a significant decline in the prevalence of total cholesterol levels exceeding $5 \mathrm{mmol} / \mathrm{l}$ between 1994 and 2006.

There is limited data on BP and cholesterol temporal trends according to medication use and few studies have formally investigated the impact of medication use on secular trends in BP and cholesterol. A report from the Minnesota Heart Survey compared the trend in total cholesterol from 1980 to 2002 among the entire population with the trend among that subgroup not using lipid-regulating medication [21]. The trends were fairly similar until the last few study years, when the general population showed a steeper decline in total cholesterol compared with the subgroup not using medication, suggesting that lipidregulating drug use may be partially responsible for the overall population-wide trend in total cholesterol at least in later years. A separate recent study in the US population found that one-quarter of an observed $0.34 \mathrm{mmol} / \mathrm{l}$ mean fall in total cholesterol between 1980 and 2000 could be attributed to statin use, in line with our findings of one-third of the (smaller) decline in non-HDL-cholesterol the UK over the same period associated with users of medication [22]. The WHO MONICA project investigated the role of medication on time trends in BP by comparing the shape of the distribution of BP in the mid-1980s with that in the mid-1990s in different worldwide populations [19]. The hypothesis was that the effect of BP-lowering medication would be realized in a selective depression of the top end of the population bell curve over time (reflecting the impact of BP-lowering medication as a high risk as opposed to mass population intervention). The study found no significant evidence for such a medication effect but may have been limited by this indirect ecological approach. 


\section{Interpretation of findings}

The marked increase in levels of the protective factor, HDL-cholesterol, which rose by about $14 \%$ between 1980 and 2000, was independent of medication use. The change is likely to reflect secular changes in the determinants of HDL-cholesterol, in particular the decline in cigarette smoking prevalence among the study population [2], as cigarette smoking is associated with markedly reduced HDL-cholesterol levels [23] and smoking cessation has been shown to raise HDL-cholesterol levels [24]. Of the more modest overall 7\% decline in non-HDL-cholesterol, approximately one-third of the decline could be accounted for by the decline occurring in users of lipid-regulating medications. The remaining population-wide decline in non-HDL-cholesterol probably reflects the contemporaneous fall in the dietary consumption of saturated fat; household purchase data suggest that daily saturated fat intake fell in the UK from 47 to $29 \mathrm{~g}$ between 1980 and 2000 [25].

The overall 5\% decline in population SBP in this survivor cohort was largely accounted for by changes among users of BP-lowering medication, reflecting the high prevalence of BPlowering medication use in this cohort of older British men. No material decline in SBP occurred among men not receiving BP-lowering medication, whereas DBP increased, which was unexpected and merits further study.

The much stronger favourable declines in non-HDL-cholesterol and SBP among medication users, compared with men not using medication, could be attributed entirely to the medication itself or could also partly reflect more favourable lifestyle changes or a differing risk profile among medication users compared with men not using medication. The persistence of differential trends according to medication use even after adjustment for concurrent changes in major potential confounders (smoking status, alcohol use, BMI and physical inactivity), and in sensitivity analyses excluding men with cardiovascular morbidity, suggests that it is most likely the treatment itself that is bringing about the changes in medication users. However, a limitation is that diet was not measured in this analysis. Medication users may also have made more favourable changes to their diet than those not using medication, which may explain some part of the differential trends. That said, although it is recognized that lipid-regulating medications act principally on non-HDLcholesterol rather than HDL, we might expect diet to have more similar influences on both lipid types. Thus, if diet did differ substantially between the two medication groups, we might have expected differences in the trends in HDL between the two groups as well, which we did not see. This merits further research.

It has been known for many years that high BP and high blood cholesterol (particularly LDL-cholesterol) are causally related to the risk of CHD. During the 1980s and 1990s, evidence on the reversibility of CHD risk associated with these factors was strengthened by the growing evidence from clinical trials [26] and guidelines for CHD prevention recommended the need to reduce BP and cholesterol levels to cut overall CHD risk [27]. In addition, the case for population-wide strategies to reduce these factors has been emphasized [28]. The results of this study indicate that only very modest progress was made in the achievement of population-wide risk factor reductions during this period, particularly when compared with the markedly greater risk factor reductions made in other populations [29]. The very modest improvements in BP and non-HDL-cholesterol may also have been influenced by the unfavourable secular increase in the prevalence of obesity, an important determinant of both BP and non-HDL-cholesterol levels.

The limited progress to date in reducing population levels of key risk factors for CHD has led to pessimism about the potential of population strategies [30], and to increasing enthusiasm for the widespread use of medications to lower risk factor levels among people without established CHD [31]. However, the high-risk approach based on medicating 
individuals has recently been heavily criticized on theoretical and experimental grounds $[32,33]$. The results of this study confirm that the use of medication to lower BP was widespread before 2000, though the precise impact of such medication use on CHD risk in the population will have depended critically on the levels of absolute risk among men receiving medications. Since 2000, further population-wide declines in blood cholesterol and BP have occurred [20]. At least part of these changes are likely to reflect further increases in the prevalence of lipid-lowering and BP-lowering drugs since 2000 [34]; the contribution of population-wide changes in the determinants of non-HDL-cholesterol and BP remains unclear, and merits urgent appraisal.

Appreciable further opportunities for the reduction in risk factor levels remain. CHD mortality risk continues to decline to $115 / 75 \mathrm{mmHg}$ for $\mathrm{BP}$ and $3.5 \mathrm{mmol} / \mathrm{l}$ for non-HDLcholesterol [35,36]. Despite the favourable declines in SBP, levels were still higher on average at the 20-year follow-up than the $140 \mathrm{mmHg}$ recommended in guidelines. Predicted DBP for a 60-year-old at the 20-year follow-up was lower than the $90 \mathrm{mmHg}$ limit for hypertension. However, given the association of DBP with type II diabetes (stronger than for SBP) [6,37], and given the alarming increase in type II diabetes in recent years [38], efforts to control the rising population DBP are also important. A recent analysis of 2006 data from the Health Survey for England suggested that, even now, BP is controlled in only about $28 \%$ of hypertensive individuals [39]. This echoes similar findings from the US population [40]. Both medication-based strategies and nonmedical population-wide strategies still have considerable potential to reduce CHD and stroke risk in the UK. Nonmedical strategies include, in particular, reduction of saturated fat and salt intakes, given that in 2007, average salt intake was estimated to be above $8.5 \mathrm{~g}$ per person per day, considerably greater than the $5.8 \mathrm{~g}$ advocated by DASH [41]. Likewise, the percentage of energy derived from saturated fat was $14.5 \%$ [42], roughly twice the desirable levels seen in Japan [43].

\section{Conclusion}

A marked increase in HDL levels has occurred in this representative cohort of older British men, which probably reflects changes in health behaviours, particularly the decline in cigarette smoking prevalence. Favourable changes in BP and non-HDL-cholesterol level have been modest and, particularly, in the case of BP, mainly reflect changes among medication users in this survivor cohort. Further efforts are needed to reduce levels of nonHDL-cholesterol and BP, through medication and population-wide changes in underlying determinants (particularly concerted changes in dietary saturated fat and salt intake).

\section{Acknowledgments}

This work was supported by the British Heart Foundation [BHF Programme grant no. RG/04/003] and Medical Research Council [training fellowship no. G0701739 to S.H.]. The British Regional Heart Study has local (from each of the districts in which the study was based) and multicentre ethics committee approvals.

\section{References}

1. British Heart Foundation Statistics Database: Coronary heart disease statistics. www.heartstats.org [Last accessed 20 September 2008]

2. Hardoon SL, Whincup PH, Lennon LT, Wannamethee SG, Capewell S, Morris RW. How much of the recent decline in the incidence of myocardial infarction in British men can be explained by changes in cardiovascular risk factors? Evidence from a prospective population-based study. Circulation. 2008; 117:598-604. [PubMed: 18212284]

3. Kuulasmaa K, Tunstall-Pedoe H, Dobson A, Fortmann S, Sans S, Tolonen H, et al. Estimation of contribution of changes in classic risk factors to trends in coronary-event rates across the WHO MONICA Project populations. Lancet. 2000; 355:675-687. [PubMed: 10703799] 
4. Capewell S, Morrison CE, McMurray JJ. Contribution of modern cardiovascular treatment and risk factor changes to the decline in coronary heart disease mortality in Scotland between 1975 and 1994. Heart. 1999; 81:380-386. [PubMed: 10092564]

5. Unal B, Critchley JA, Capewell S. Explaining the decline in coronary heart disease mortality in England and Wales between 1981 and 2000. Circulation. 2004; 109:1101-1107. [PubMed: 14993137]

6. Mancia G, De Backer G, Dominiczak A, Cifkova R, Fagard R, Germano G, et al. 2007 Guidelines for the management of arterial hypertension: The Task Force for the Management of Arterial Hypertension of the European Society of Hypertension (ESH) and of the European Society of Cardiology (ESC). Eur Heart J. 2007; 28:1462-1536. [PubMed: 17562668]

7. Carleton RA, Dwyer J, Finberg L, Flora J, Goodman DS, Grundy SM, et al. Report of the expert panel on population strategies for blood cholesterol reduction. A statement from the National Cholesterol Education Program, National Heart, Lung, and Blood Institute, National Institutes of Health. Circulation. 1991; 83:2154-2232. [PubMed: 2040066]

8. Office for National Statistics. Prescription cost analysis 2007. The Information Centre; Leeds: 2008.

9. Walker M, Whincup PH, Shaper AG. The British Regional Heart Study 1975-2004. Int J Epidemiol. 2004; 33:1185-1192. [PubMed: 15319395]

10. Whincup PH, Bruce NG, Cook DG, Shaper AG. The Dinamap 1846SX automated blood pressure recorder: comparison with the Hawksley random zero sphygmomanometer under field conditions. J Epidemiol Community Health. 1992; 46:164-169. [PubMed: 1583434]

11. Wannamethee SG, Shaper AG. Taking up regular drinking in middle age: effect on major coronary heart disease events and mortality. Heart. 2002; 87:32-36. [PubMed: 11751661]

12. Shaper AG, Wannamethee G, Weatherall R. Physical activity and ischaemic heart disease in middle-aged British men. Heart. 1991; 66:384-394. [PubMed: 1747302]

13. Office for National Statistics. Key health statistics from general practice 1998. Crown; UK: 2000.

14. Klungel OH, de Boer A, Paes AH, Herings RM, Seidell JC, Bakker A. Agreement between selfreported antihypertensive drug use and pharmacy records in a population-based study in The Netherlands. Pharm World Sci. 1999; 21:217-220. [PubMed: 10550846]

15. Thomas MC, Walker M, Lennon LT, Thomson AG, Lampe FC, Shaper AG, et al. Non-attendance at re-examination 20 years after screening in the British Regional Heart Study. J Public Health Med. 2002; 24:285-291. [PubMed: 12546205]

16. Barnett AG, van der Pols JC, Dobson AJ. Regression to the mean: what it is and how to deal with it. Int J Epidemiol. 2005; 34:215-220. [PubMed: 15333621]

17. Evans, B.; Primatesta, P. Health Survey for England 1998. The Stationary Office; London: 1999.

18. Antikainen RL, Moltchanov VA, Chukwuma C Sr, Kuulasmaa KA, Marques-Vidal PM, et al. Trends in the prevalence, awareness, treatment and control of hypertension: the WHO MONICA Project. Eur J Cardiovasc Prev Rehabil. 2006; 13:13-29. [PubMed: 16449860]

19. Tunstall-Pedoe H, Connaghan J, Woodward M, Tolonen H, Kuulasmaa K. Pattern of declining blood pressure across replicate population surveys of the WHO MONICA project, mid-1980s to mid-1990s, and the role of medication. BMJ. 2006; 332:629-635. [PubMed: 16531419]

20. Joint Health Surveys Unit. Health Survey for England 2006. Cardiovascular disease and risk factors. The Information Centre; Leeds: 2008.

21. Arnett DK, Jacobs DR Jr, Luepker RV, Blackburn H, Armstrong C, Claas SA. Twenty-year trends in serum cholesterol, hypercholesterolemia, and cholesterol medication use: the Minnesota Heart Survey, 1980-1982 to 2000-2002. Circulation. 2005; 112:3884-3891. [PubMed: 16344385]

22. Young, F.; Critchley, JA.; Capewell, S. Attribution of coronary mortality declines in the USA between 1980 and 2000 to primary and secondary prevention. Euro PRevent 2009 conference; Stockholm. May 2009; [Abstract 543]

23. Campbell SC, Moffatt RJ, Stamford BA. Smoking and smoking cessation-The relationship between cardiovascular disease and lipoprotein metabolism: a review. Atherosclerosis. 2008; 201:225-235. [PubMed: 18565528]

24. Hausenloy DJ, Yellon DM. Targeting residual cardiovascular risk: raising high-density lipoprotein cholesterol levels. Heart. 2008; 94:706-714. [PubMed: 18480348] 
25. National Food Survey 2000. https://statistics.defra.gov.uk/esg/publications/nfs/2000 [Last Accessed 27 March 2009]

26. Baigent C, Keech A, Kearney PM, Blackwell L, Buck G, Pollicino C, et al. Efficacy and safety of cholesterol-lowering treatment: prospective meta-analysis of data from 90056 participants in 14 randomised trials of statins. Lancet. 2005; 366:1267-1278. [PubMed: 16214597]

27. De Boer G, Ambrosioni E, Borch-Johnsen K, Brotons C, Cifkova R, Dallongeville J, et al. European guidelines on cardiovascular disease prevention in clinical practice: third joint task force of European and other societies on cardiovascular disease prevention in clinical practice (constituted by representatives of eight societies and by invited experts). Eur J Cardiovasc Prev Rehabil. 2003; 10:S1-S10. [PubMed: 14555889]

28. Rose G. Strategy of prevention: lessons from cardiovascular disease. BMJ. 1981; 282:1847-1851. [PubMed: 6786649]

29. Uusitalo U, Feskens EJM, Tuomilehto J, Dowse G, Haw U, Fareed D, et al. Fall in total cholesterol concentration over five years in association with changes in fatty acid composition of cooking oil in Mauritius: cross sectional survey. BMJ. 1996; 313:1044-1046. [PubMed: 8898594]

30. Manuel DG, Lim J, Tanuseputro P, Anderson GM, Alter DA, Laupacis A, et al. Revisiting Rose: strategies for reducing coronary heart disease. BMJ. 2006; 332:659-662. [PubMed: 16543339]

31. Wald NJ, Law MR. A strategy to reduce cardiovascular disease by more than $80 \%$. BMJ. 2003; 326:1419. [PubMed: 12829553]

32. Rose, GA. The strategy of preventive medicine. Oxford University Press; Oxford: 1992.

33. Emberson J, Whincup P, Morris R, Walker M, Ebrahim S. Evaluating the impact of population and high-risk strategies for the primary prevention of cardiovascular disease. Eur Heart J. 2004; 25:484-491. [PubMed: 15039128]

34. DeWilde S, Carey IM, Richards N, Whincup PH, Cook DG. Trends in secondary prevention of ischaemic heart disease in the UK 1994-2005: use of individual and combination treatment. Heart. 2008; 94:83-88. [PubMed: 17540684]

35. Lewington S, Clarke R, Qizilbash N, Peto R, Collins R. Age-specific relevance of usual blood pressure to vascular mortality: a meta-analysis of individual data for one million adults in 61 prospective studies. Lancet. 2002; 360:1903-1913. [PubMed: 12493255]

36. Lewington S, Whitlock G, Clarke R, Sherliker P, Emberson J, Halsey J, et al. Blood cholesterol and vascular mortality by age, sex, and blood pressure: a meta-analysis of individual data from 61 prospective studies with 55000 vascular deaths. Lancet. 2007; 370:1829-1839. [PubMed: 18061058]

37. Zanchetti A, Ruilope LM. Antihypertensive treatment in patients with type-2 diabetes mellitus: what guidance from recent controlled randomized trials? J Hypertens. 2002; 20:2099-2110. [PubMed: 12409940]

38. National Centre for Social Research: Health Survery for England 2006. Adult trend tables. http:// www.ic.nhs.uk/webfiles/publications/HSE06/ [Last Accessed 4 December 2008]

39. Falaschetti E, Chaudhury M, Mindell J, Poulter N. Continued improvement in hypertension management in England: results from the Health Survey for England 2006. Hypertension. 2009; 53:480-486. [PubMed: 19204180]

40. Ong KL, Cheung BM, Man YB, Lau CP, Lam KS. Prevalence, awareness, treatment, and control of hypertension among United States adults 1999-2004. Hypertension. 2007; 49:69-75. [PubMed: 17159087]

41. Sacks FM, Svetkey LP, Vollmer WM, Appel LJ, Bray GA, Harsha D, et al. DASH-Sodium Collaborative Research Group. Effects on blood pressure of reduced dietary sodium and the Dietary Approaches to Stop Hypertension (DASH) diet. N Engl J Med. 2001; 344:3-10. [PubMed: 11136953]

42. Department of Environment, Farming and Rural Affairs. Family food in 2007. Crown; London: 2008.

43. Ueshima H, Stamler J, Elliott P, Chan Q, Brown IJ, Carnethon MR, et al. Food omega-3 fatty acid intake of individuals (total, linolenic acid, long-chain) and their blood pressure: INTERMAP study. Hypertension. 2007; 50:313-319. [PubMed: 17548718] 


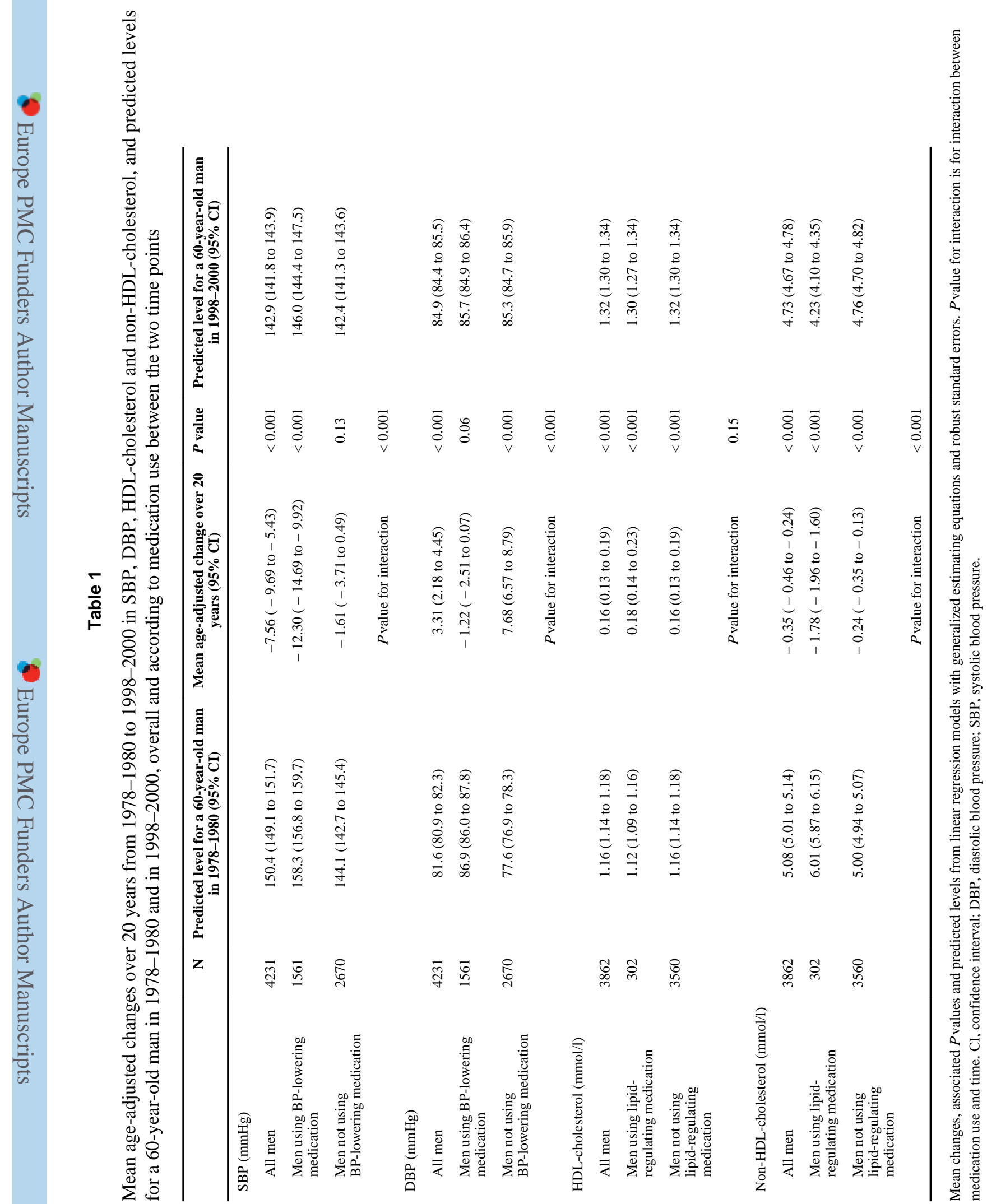

